\title{
THE PROJECT PLASMONX FOR PLASMA ACCELERATION EXPERIMENTS AND A THOMSON X-RAY SOURCE AT SPARC
}

\author{
D. Alesini, M. Bellaveglia, S. Bertolucci, M.E. Biagini, R. Boni, M. Boscolo, M. Castellano,
} A. Clozza, G. Di Pirro, A. Drago, A. Esposito, M. Ferrario, L. Ficcadenti, D. Filippetto, V. Fusco, A. Gallo, G. Gatti, A. Ghigo, S. Guiducci, M. Incurvati, C. Ligi, F. Marcellini, M. Migliorati, A. Mostacci, L. Palumbo, L. Pellegrino, M. Preger, R. Ricci, C. Sanelli, M. Serio, F. Sgamma, B. Spataro, A. Stecchi, A. Stella, F. Tazzioli, C. Vaccarezza, M. Vescovi, C. Vicario, INFN-LNF, Frascati, Italy

F. Alessandria, A. Bacci, R. Bonifacio, I. Boscolo, F. Broggi, S. Cialdi, C. DeMartinis, D. Giove, C. Maroli, M. Mauri, V. Petrillo, N. Piovella, R. Pozzoli, M. Romè, L. Serafini*, INFN-MI and Univ. of Milano, Milano, Italy

M. Mattioli, P. Musumeci, M. Petrarca, INFN-Romal, Rome, Italy

U. Bottigli, B. Golosio, P. Oliva, A. Poggiu, S. Stumbo, INFN/Cagliari and Univ. of Sassari, Italy

A. Barbini, W. Baldeschi, C.A. Cecchetti, M. Galimberti, A. Giulietti, D. Giulietti, L.A. Gizzi, P. Koester, L. Labate, S. Laville, A. Rossi, P. Tomassini, CNR-IPCF/Pisa and Univ. of Pisa, Italy.

\section{Abstract}

We present the status of the project PLASMONX, recently approved by INFN. This project, based on a collaboration between INFN and CNR-IPCF, aims at a long term upgrade of the SPARC system with the goal to develop at LNF an integrated facility for advanced beamlaser-plasma research in the field of advanced acceleration techniques and ultra-bright $\mathrm{X}$-ray radiation sources and related applications. The project, in its first phase, foresees the development at LNF of a High Intensity Laser Laboratory (HILL) whose main component is a 100 TW-class Ti:Sa laser system synchronized to the SPARC photo-injector. Experiments of self-injection and acceleration of electrons into laser driven plasma waves will be conducted at HILL-LNF, early in this first project phase. Eventually an additional beam line will be built in the SPARC bunker in order to transport the SPARC electron beam at an interaction point, where a final focus system will allow to conduct experiments either of laser-beam co-propagation in plasma waves for high gradient acceleration, or experiments of laser-beam head-on collisions to develop a Thomson source of bright ultra-short $\mathrm{X}$-ray radiation pulses, with X-ray energies tunable in the range 20 to $1000 \mathrm{keV}$ and pulse duration from 30 fs to $20 \mathrm{ps}$. Preliminary simulations of plasma acceleration with selfinjection are illustrated, as well as external injection of the SPARC electron beam.

\section{DEVELOPMENT OF A 100 TW-CLASS LASER SYSTEM AT LNF}

While the SPARC Project is in progress at LNF aiming at the construction by the year 2006 of an advanced photoinjector driving a FEL[1], its long term upgrade, based on the project PLASMONX, has just been launched after approval by INFN, with a first phase aimed at building at LNF a $100 \mathrm{TW}$-class laser system as the core system of a High Intensity Laser Laboratory (HILL), and a final goal of constituting a national facility merging advanced technologies and expertises in high brightness electron beams and high intensity laser beams, as well as plasma wave formation, control and diagnostics. Under this respect, the collaboration between INFN and CNRIPCF is considered to be strategic to the realization of the final phase of the facility, where synchronized electron and photon beams of ultra-high performances, in terms of brightness, intensity and brevity will be driven to interact in several fashions, in order to allow investigations of high gradient acceleration techniques and/or X-ray production via Thomson back-scattering in the spontaneous incoherent mode or, eventually, in the coherent collective mode. The proposed time schedule for this initiative is tightly correlated with the progress of the SPARC project: according to this schedule, final completion of the SPARC\&PLASMONX facility is foreseen by the year 2009 .

The proposed laser system[2] must have unique performances in terms of power, pulse duration, flexibility and reliability: it will be installed inside the dedicated HILL building at LNF, presently under design, located just on top of the SPARC bunker. The laser system, based upon the C.P.A. technique, will deliver < $50 \mathrm{fs}, 800 \mathrm{~nm},>100 \mathrm{TW}$, laser pulses at $10 \mathrm{~Hz}$ rep. rate. The $5 \mathrm{~J}$ laser pulses will be transported uncompressed from the HILL laboratory down into the SPARC bunker. The proposed system combines the reliability of established Ti:Sa technology with novel additional devices aimed at overcoming known issues typical of large Ti:Sa systems."

\footnotetext{
*Luca.Serafini@mi.infn.it
} 


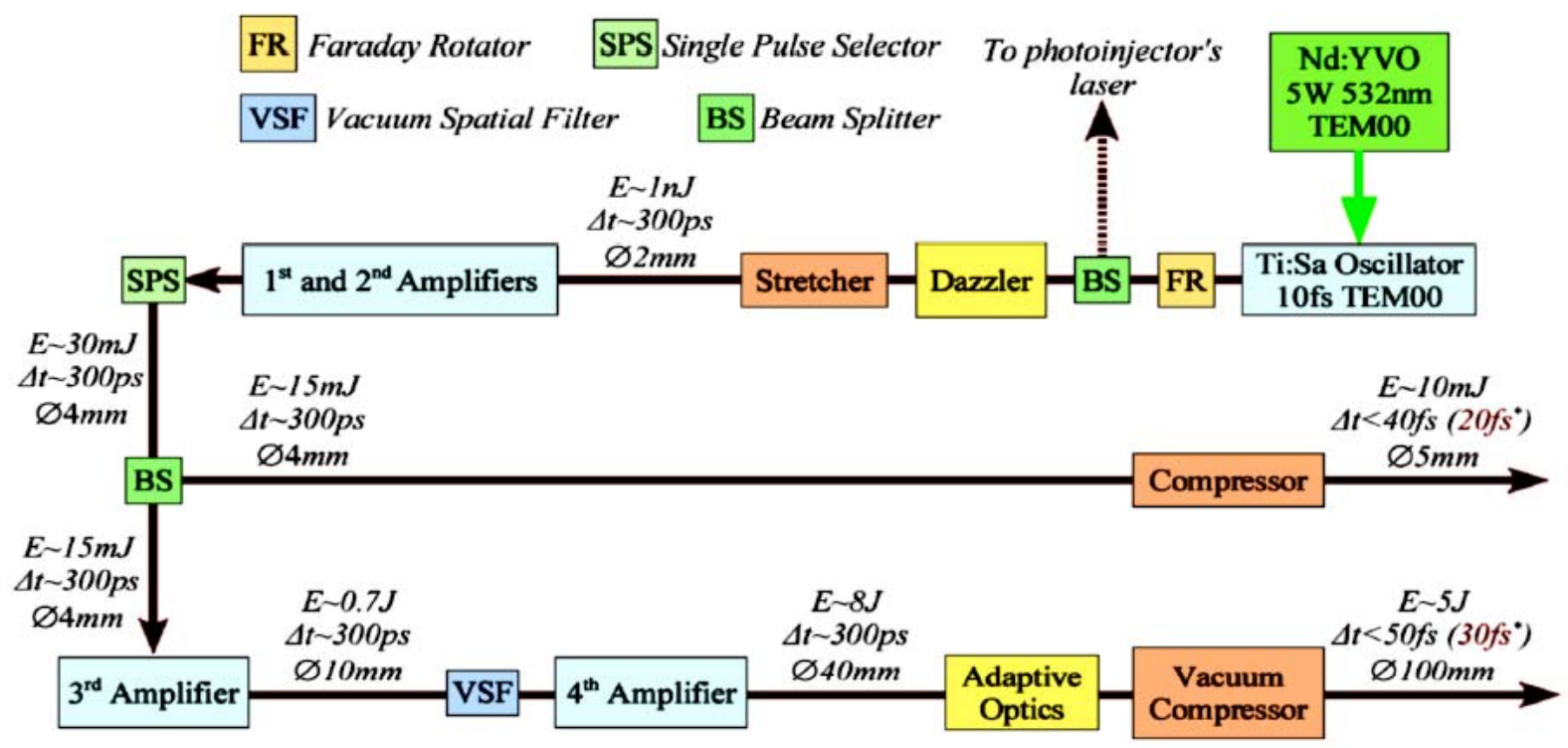

Figure 1: Block diagram of the PLASMON-X laser system showing the main functional components needed for the amplification of the stretched oscillator pulse, its compression and control of spectral and spatial features of the pulse.

One of these issues concerns the power contrast ratio, namely the ratio between the main CPA pulse and the socalled "pedestal", a spurious precursor radiation arising from amplified spontaneous emission (ASE) by amplifiers. The block diagram of Figure 1 shows the main components of the system, including the devices necessary for ensuring the high quality of the final output.

The oscillator produces a $10 \mathrm{fs}$ pulse that is stretched and pre-amplified by a two stage amplifier up to the 30 $\mathrm{mJ}$ level per pulse. Approximately half of this energy is extracted and compressed to provide a low energy beam for probing/diagnostic purposes. The remaining part of the pulse is further amplified to the $8 \mathrm{~J}$ level and finally compressed under vacuum. Besides the standard vacuum spatial filter necessary for beam cleaning, we plan to insert an adaptive optics before the vacuum compressor. This device will enable us to remove aberrations on the beam which may result in a poor performance of the compressor as well as poor quality of the focal spot after focusing. Concerning the control of the pulse duration, the system includes a Dazzler device which will enable correction of the spectral features of the pulse prior to stretching. These corrections are necessary in order to keep the final pulse-length well below the $100 \mathrm{fs}$ level. In fact, we aim at reaching the $<50$ fs pulse-length which is now regarded as the minimum pulse duration achievable in a multi-joule Ti:Sa laser system. The main beam characteristics after the vacuum compressor are foreseen to be: pulse energy $5 \mathrm{~J}$, peak power $>100 \mathrm{TW}$, contrast ratio $<10^{-5}$.

We plan to conduct R\&D on the OPCPA technique (Optical Parametric Chirped Pulse Amplification), that exploits the non-linear properties of some crystals for the amplification of optical pulses: we aim at an inherently low ASE system in which the ASE level is drastically reduced in the initial amplifier stages. In fact, it is estimated that with an OPCPA system in place, the final pulse length could be as short as $30 \mathrm{fs}$, thus leading to a peak power as high as $170 \mathrm{TW}$ on target and a contrast ratio smaller than $10^{-8}$.

Most of the proposed programme relies on the synchronisation of the laser system with the SPARC Linac and, in particular, with the photoinjector laser system. This can be done either using an electro-optics based approach or optically. In the first way the laser oscillator is synchronized with an external rf signal, by changing dynamically the oscillator cavity length: a jitter between the rf and the laser pulse of less than 1ps is typically achieved. The fully optical approach consists in synchronising the two laser systems. This can be done either by using the same oscillator for both lasers or by measuring the change in delay between the two laser pulses (i.e. using a single shot second order cross correlator) with a precision of tens of femtoseconds, and then adjust dynamically a delay line in one of the two laser system.

\section{PLASMA ACCELERATION EXPERIMENTS}

Here we describe two possible plasma acceleration experiments that can be performed at HILL (first phase) and inside the SPARC bunker (final phase, external injection).

\section{Self-injection}

Following a scheme proposed by Bulanov et al.[3], we studied by simulations the self-injection of electrons trapped by a plasma wave driven across a density transition, as depicted in Fig.2. A 17 fs (FWHM) laser pulse of intensity $\mathrm{I}=2.5 \cdot 10^{18} \mathrm{~W} / \mathrm{cm}^{2}$ is focused to a $20 \mu \mathrm{m}$ 
waist into the plasma: a bunch of $10^{8}$ electrons with energy $\mathrm{E} \approx 10 \mathrm{MeV}$ is generated, with small energy spread $\Delta \mathrm{T} / \mathrm{T} \approx 5 \%$, very short bunch length, $\sigma_{\mathrm{z}} \approx 5 \mu \mathrm{m}$, and quite low transverse emittance, $0.1 \mathrm{~mm} \cdot \mathrm{mrad}$.

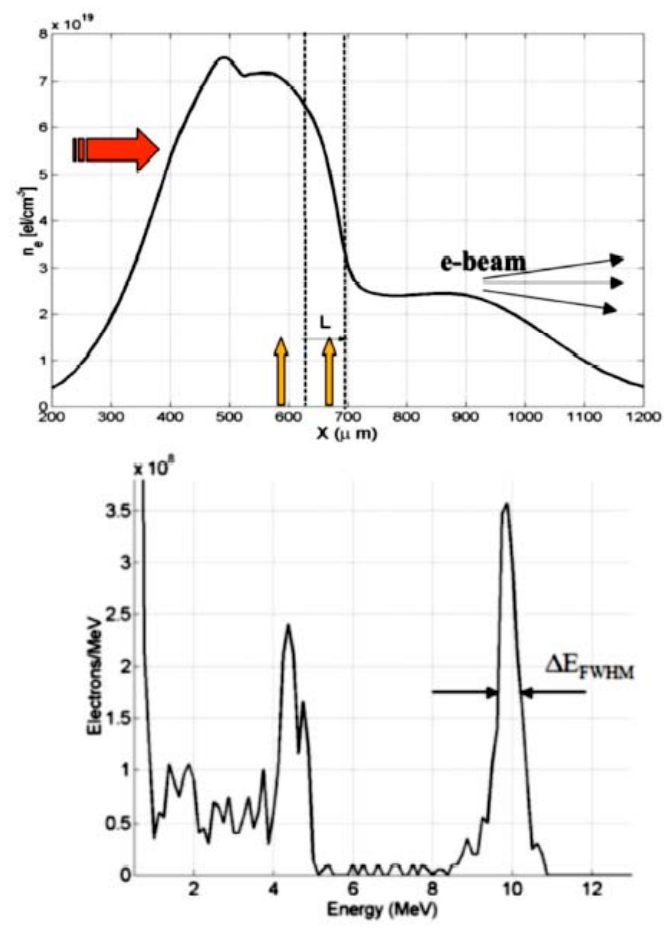

Figure 2 - (lower diagram) Energy spectrum of the accelerated electrons in a plasma with sharp density depletion (upper diagram), generated by 2-d hydrodynamic simulations of an exploding two-foil target (marked by yellow arrows). Red arrow shows the laser pulse propagation.

\section{External Injection}

A numerical experiment of injecting an electron bunch produced by the SPARC Linac into a plasma is based on a simulation technique using a test-particle code that assumes a quasi-static field approximation. Transverse fields are at least two orders of magnitude less than the longitudinal ones and are safely neglected, while nonlinear effects are treated in a 2-d representation. The injected electron bunch has a charge of $21 \mathrm{pC}$ at an energy of $130 \mathrm{MeV}$, with $8 \mu \mathrm{m}$ (rms) transverse size and rms length of $\sigma_{z}=12 \mu \mathrm{m}$. The energy spread is about $0.1 \%$. The electron bunch has been obtained by a start-toend simulation of the beam dynamics in the SPARC photo-injector, and transported through the PLASMONX beam-line to the interaction point[4]. The simulation uses a 4J laser pulse with $50 \mathrm{fs}$ pulse length at $800 \mathrm{~nm}$ wavelength, focused in a $50 \mu \mathrm{m}$ spot into a preformed plasma of density of $5 \times 10^{16} \mathrm{~cm}^{-3}$. The bunch has a longitudinal extent which is small but not negligible compared to the wavelength of the plasma wave. The energy distribution of the electrons at the exit of the plasma is shown in Figure 3 for two different plasma acceleration lengths of $20 \mathrm{~mm}$ and $100 \mathrm{~mm}$ (this implies guiding the laser pulse into a plasma channel [2] or a capillary discharge).

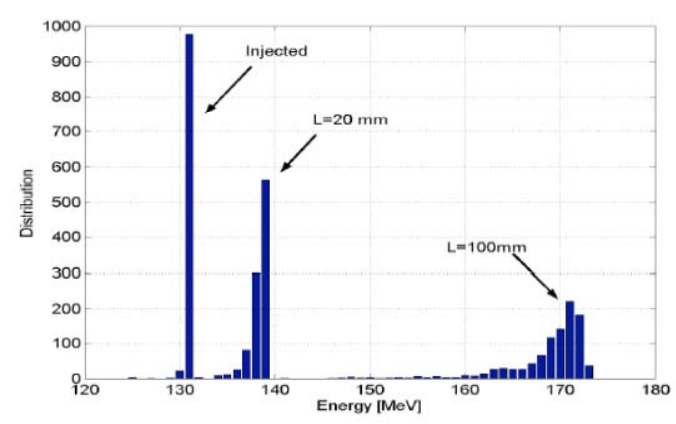

Figure 3 - Electron energy distribution at the exit of a plasma of $5 \times 10^{16} \mathrm{~cm}^{-3}$ for an acceleration length of 20 $\mathrm{mm}$, corresponding to twice the Rayleigh length $Z_{R}$ of the focusing optics. Also shown in the plot is the result of an accelerator length of $100 \mathrm{~mm}$, assuming a laser pulse guiding over $5 \mathrm{Z}_{\mathrm{R}}$. The energy spread of the output electrons is $3 \%(\mathrm{rms})$.

\section{X-RAY THOMSON SOURCE}

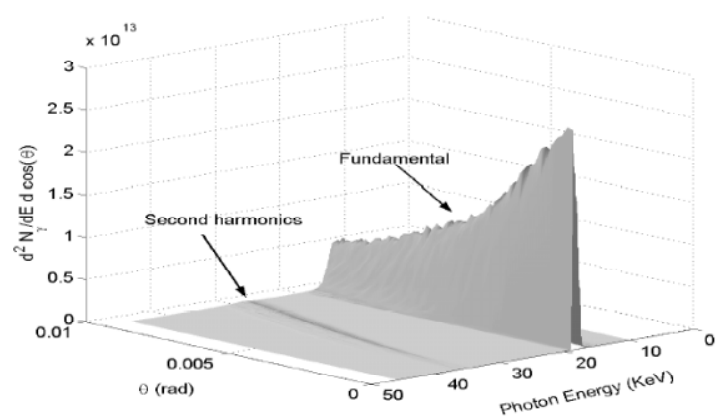

Figure 4 - Angular and spectral distribution of the Thomson source radiation: unguided 3 ps laser pulse focused to $12.5 \mu \mathrm{m}$ beam waist size

By colliding in vacuum the SPARC electron beam[4] and the HILL laser pulse ( $3 \mathrm{ps}, 4 \mathrm{~J}$ ) we evaluated[5] a yield of $9.3 \cdot 10^{8}$ photons/s at a tunable energy in the 20$1000 \mathrm{keV}$ range, with angular and spectral distribution as shown in Fig.4. By guiding the laser pulse one can upgrade the photon yield per pulse up to $1.6 \cdot 10^{9}$.

\section{REFERENCES}

[1] D. Alesini et al., NIM A528 (2004) 586

D. Alesini et al., Status of the SPARC Project, these proceedings

[2] D. Giulietti et al., PLASMONX: Conceptual Design Report on PLASma acceleration and MONochromatic $X$-ray production, unpublished

[3] S.V. Bulanov et al., Phys. Rev. E 58 (5) R5257

[4] D. Alesini et al., Design Study for Advanced Acceleration Experiments and Monochromatic X-ray Production@SPARC, Proc. EPAC-2004,pag. 695, ISBN: 92-9083-231-2A

[5] P. Tomassini et al, Appl. Phys. B 89 419-436 (2005) 